\title{
Comparison of two sperm processing techniques for low complex- ity assisted fertilization: sperm washing followed by swim-up and discontinuous density gradient centrifugation
}

\author{
Cássio L Fácio ${ }^{1}$, Lígia F Previato ${ }^{1}$, Ligiane A Machado-Paula ${ }^{1}$, Paulo CS Matheus ${ }^{1}$, Edilberto Araújo Filho ${ }^{1}$ \\ ${ }^{1}$ Center of Human Reproduction of São Jose do Rio Preto - São José do Rio Preto - SP, Brazil
}

Presented at the XXV Brazilian Congress of Human Reproduction, São Paulo, SP, Brazil, November, 2012.

\begin{abstract}
Objective: This study aimed to assess and compare sperm motility, concentration, and morphology recovery rates, before and after processing through sperm washing followed by swim-up or discontinuous density gradient centrifugation in normospermic individuals.

Methods: Fifty-eight semen samples were used in double intrauterine insemination procedures; 17 samples (group 1) were prepared with sperm washing followed by swim-up, and 41 (group 2) by discontinuous density gradient centrifugation. This prospective non-randomized study assessed seminal parameters before and after semen processing. A dependent t-test was used for the same technique to analyze seminal parameters before and after semen processing; an independent t-test was used to compare the results before and after processing for both techniques.

Results: The two techniques produced decreases in sample concentration (sperm washing followed by swim-up: $P<0.000006$; discontinuous density gradient centrifugation: $P=0.008457)$ and increases in motility and normal morphology sperm rates after processing. The difference in sperm motility between the two techniques was not statistically significant. Sperm washing followed by swim-up had better morphology recovery rates than discontinuous density gradient centrifugation $(P=0.0095)$; and the density gradient group had better concentration recovery rates than the swim-up group ( $P=0.0027)$.

Conclusion: The two methods successfully recovered the minimum sperm values needed to perform intrauterine insemination. Sperm washing followed by swim-up is indicated for semen with high sperm concentration and better morphology recovery rates. Discontinuous density gradient centrifugation produced improved concentration recovery rates.
\end{abstract}

Keywords: Intrauterine insemination, semen processing, swim-up, discontinuous concentration gradient.

\section{INTRODUCTION}

Spermatozoa undergo a series of biochemical and structural changes within the female genital tract called capacitation (Neves, 1991). In-vivo, capacitation occurs over a period of seven hours (Yoshida et al., 2008). During this period, the glycoprotein coat and seminal proteins are removed from the surface of the sperm's acrosome. Capacitated spermatozoa show highly active flagellar beating (hyperactivation), undergo the acrosome reaction, penetrate the pellucid zone, and finally bind and fuse with the oocytes (Yoshida et al., 2008).

Sperm capacitation in assisted human reproduction (AHR) is performed artificially using specific techniques. Intrauterine insemination (IUI), an assisted reproduction technology ( $A R T)$, is a non-invasive method and the least expensive of ARTs (Dodson \& Haney,
1991). Several factors affect the success rates of IUI; severe male, tubal, and peritoneal factors and severe endometriosis had to be excluded (Scemama et al. 1995). Seminal parameters also significantly impact the outcome of IUI (Arny \& Quagliarello, 1987; Brasch et al. 1994; Berg et al., 1997; Dorjpurev et al., 2011).

Sperm processing techniques for IUI vary from laboratory to laboratory and even from patient to patient (Zhao et al., 2004). Furthermore, these techniques have become very useful in the treatment of male infertility, allowing for better sperm recovery and improvements in sperm motility rates (Barroso, et al., 2005).

The purpose of semen processing is to increase the concentration of motile sperm, and to remove seminal plasma, debris, prostaglandins, and other substances deleterious for sperm viability that cause uterine contractions and bacterial contamination (Pasqualotto, 2007). Removal of immotile sperm, leukocytes, or immature germ cells is another advantage of these techniques, and one that can be an important factor for increasing seminal quality (Aitken \& Clarkson, 1987). Therefore, sperm used in IUI must be processed (i.e., separated) from the seminal fluid, capacitated, and then selected based on morphology and motility to be able to be introduced into the uterine cavity (Huang et al., 1996).

The swim-up technique is easy to perform, cost-effective, and usually recovers a very clean fraction of highly motile spermatozoa (Henkel \& Schill, 2003).

Sperm processing by the discontinuous concentration gradient technique usually recovers a clean fraction of highly motile spermatozoa, thus allowing the separation of spermatozoa from ejaculates with very low sperm density and providing good yield, largely eliminating leukocytes and significantly reducing reactive oxygen species (Henkel \& Schill, 2003).

This study aimed to assess and compare sperm motility, concentration, and morphology recovery rates, before and after processing through sperm washing followed by swim-up or discontinuous density gradient centrifugation in normospermic individuals.

\section{MATERIAL AND METHODS}

Fifty-eight semen samples from 17 couples submitted to double homologous IUI cycles from 2008 to 2010 in the Center for Human Reproduction of São José do Rio Preto, $\mathrm{SP}$, Brazil, were included in this prospective non-randomized study. Some patients underwent IUI in more than one reproductive cycle.

The individuals enrolled in the study met the following inclusion criteria: male patients were normozoospermic; female patients were aged 38 years or younger, had mild endometriosis, and cervical factor infertility with at least one normal fallopian tube as determined by hysterosalpingography or video laparoscopy. Exclusion criteria included 
severe endometriosis, tubal-peritoneal factor, and male factor infertility. Enrolled couples signed informed consent terms before joining the study.

The 58 semen samples were divided into two groups: group 1 with 17 samples submitted to sperm washing followed by swim-up; and group 2, with 41 samples submitted to discontinuous density gradient centrifugation.

Semen parameters (concentration, motility, and morphology) were used in the choice of technique. Samples with sperm concentrations below 20 million cells $/ \mathrm{ml}$, sperm motility equal to or greater than $50 \%$, and patients with previous failed IUI procedures were excluded from the swim-up group and included in the discontinuous density gradient centrifugation group.

\section{Ovarian stimulation protocol}

All patients underwent ovarian stimulation with 100 $\mathrm{mg} /$ day of clomiphene citrate from days 3 to 7 of the menstrual cycle, along with subcutaneous human menopausal gonadotropin (hMG, 75 IU) (Menopur; Ferring Ltda, Brazil) at days 3, 5, and 7 of the cycle.

Transvaginal ultrasound (baseline) (Midray-Expert $3 \mathrm{C} 5 \mathrm{~A}$; China) was performed on day 2 of the cycle for all patients before ovarian hyperstimulation. Follicular development was monitored by transvaginal ultrasound starting on day 8 or 9 of the cycle and later depending on each case; and when at least one follicle reached $20 \mathrm{~mm}$ in diameter, 5000 IU of human chorionic gonadotropin (hCG) (Ovidrel, Serono, Brazil) were administered subcutaneously. IUI was scheduled 36 to 40 hours after hCG once ovulation was confirmed.

\section{Semen analysis and capacitation}

Semen samples were collected by masturbation after 3-5 days of sexual abstinence. The seminal parameters were analyzed by only one observer and categorized according to the 1999 WHO criteria (WHO, 1999): complete liquefaction within 60 minutes; sperm concentration (M/ $\mathrm{ml}) \geq 20$ million; motility (grade $\mathrm{A}+\mathrm{B}$ ) $>50 \%$; morphology $\geq 14 \%$.

Seventeen semen samples from group 1 were processed through sperm washing followed by swim-up. First, the sample was washed with modified human tubal fluid (mHTF) medium with HEPES (Irvine Scientific), supplemented with $15 \%$ synthetic serum substitute (SSS, Irvine Scientific), and centrifuged at $270 \times \mathrm{g}$ for 5 minutes (Excelsa Baby I - Model 206, FANEN, São Paulo, Brazil); the supernatant was subsequently removed. After that, the swim-up technique was performed, in which the pellet was resuspended and homogenized in modified human tubal fluid (mHTF) medium with HEPES until a volume of $1 \mathrm{ml}$ was reached. The resuspended pellet was removed with a Pasteur pipette and added into another $15 \mathrm{ml}$ conical Falcon tube containing $1 \mathrm{ml}$ of modified human tubal fluid (mHTF) medium with HEPES (care was taken so that both did not mix). The tube was inclined at an angle of 450 and incubated for 50 minutes at $370 \mathrm{C}$. Then, $0.5 \mathrm{ml}$ was removed from the surface of the supernatant to perform post-processing seminal parameter analysis.

Forty-one semen samples from group 2 were processed using the discontinuous density gradient centrifugation method using the Isolate stock (Irvine Scientific, Santa Ana, CA, USA). The total volume of semen was divided so that a $15-\mathrm{ml}$ conical Falcon tube contained $1 \mathrm{ml}$ of a $90 \%$ density lower layer, $1 \mathrm{ml}$ of a $50 \%$ density upper layer, and $1 \mathrm{ml}$ of semen (1/1/1). The sample was then centrifuged at $270 \times \mathrm{g}$ for 15 minutes (Excelsa Baby I - Model 206, FANEN, São Paulo, Brazil).

After centrifugation, the supernatant was removed and the spermatozoa (pellet) was placed into another 15$\mathrm{ml}$ Falcon tube, which contained $5 \mathrm{ml}$ of modified human tubal fluid (mHTF) medium with HEPES (Irvine Scientific), supplemented with $15 \%$ synthetic serum substitute (SSS, Irvine Scientific); it was then centrifuged at $270 \times \mathrm{g}$ for 10 minutes. The final pellet was resuspended in the same medium solution, obtaining a final volume of $1 \mathrm{ml}$. A $10-\mu \mathrm{l}$ aliquot was used to perform the analysis of post-processing seminal parameters.

\section{Statistical analysis}

A dependent t-test was performed for group 1 samples comparing semen parameters before and after semen processing by sperm washing followed by the swim-up technique; for group 2, the comparison involved semen parameters before and after semen processing by the discontinuous density gradient centrifugation technique.

An independent t-test was performed to compare the seminal parameters of samples in groups 1 and 2 at two different times: (i) before semen processing and (ii) after semen processing.

Data analysis was carried out using the SAS 9.3 System for Windows; differences yielding $p$-values of $5 \%$ or lower were deemed statistically significant.

\section{RESULTS}

The assessment of each technique in terms of semen parameters before and after processing revealed that both resulted in decreased sperm concentration after processing $(P=0.000006$ for sperm washing followed by swim-up; and $P=0.008457$ for discontinuous density gradient centrifugation), and increased motility and rates of morphologically normal sperm $(P=0.00001$ for sperm washing followed by swim-up; and $P<0.05$ for discontinuous density gradient centrifugation) after processing (Tables 1 and 2).

However, when the results before processing of both techniques were compared, no statistically significant differences were found in regards to sperm concentration, motility, or morphology (Table 3).

Unlike motility $(P=0.9825)$, the differences in semen concentration $(P=0.0027)$ and morphology (normal $P=0.0095$; abnormal $P=0.0106$ ) between the two techniques were statistically significant. In other words, the sperm concentration recovery rates seen in discontinuous density gradient centrifugation $(64.8 \pm 43.3)$ were higher than the rates observed in sperm washing followed by swim-up (29.0 \pm 28.2$)(P=0.0027)$, whereas morphology recovery rates were better in sperm washing followed by swim-up (56.7 \pm 12.8$)$ than in discontinuous density gradient centrifugation $(45.3 \pm 15.2)(P=0.0095)$ (Table 3$)$.

\section{DISCUSSION}

Swim-up and discontinuous density gradient are the most commonly used semen preparation techniques in AHR laboratories (Paasch et al., 2007; Jayaraman et al., 2012).

When used in IUI, these techniques are expected to provide at least five million motile spermatozoa. This number of sperm cells is required so that fertilization may occur in the fallopian tube. Concentrations below this limit suggest the choice of other AHR methods such as in-vitro fertilization (IVF) (Khalil et al., 2001; Wainer et al., 2004). The choice of method is based on the baseline quality of the semen sample. According to the WHO (WHO, 1999), either technique can be used for normal semen (Wainer et al., 2004).

Few studies in the literature have compared the swim-up and discontinuous density gradient techniques, and different conclusions have been proposed about them (Carrell et al., 1998; Dodson et al., 1998; Posada et al., 2005). In a meta-analysis investigating different semen preparation techniques used for IUI, the results were qualitatively and quantitatively inconclusive, as the data were insufficient to conclude 
Table 1. Comparison of seminal parameters before and after semen processing through sperm washing followed by swimup.

\begin{tabular}{|c|c|c|c|}
\hline $\begin{array}{l}\text { Sperm washing followed by swim } \\
\text { up }(n=17)\end{array}$ & $\begin{array}{c}\text { Before semen processing } \\
\text { Mean } \pm \text { Std Dev }\end{array}$ & $\begin{array}{l}\text { After semen processing } \\
\text { Mean } \pm \text { Std Dev }\end{array}$ & $P$-value * \\
\hline Concentration $\left(\times 10^{6} / \mathrm{ml}\right)$ & $92.5 \pm 56.9$ & $29.0 \pm 28.2$ & 0.000006 \\
\hline Motility (\%) & $52.8 \pm 12.9$ & $76.5 \pm 18.5$ & 0.000001 \\
\hline $\begin{array}{l}\text { Morphology (\%) } \\
\text { normal } \\
\text { abnormal }\end{array}$ & $\begin{array}{l}29.8 \pm 7.0 \\
70.1 \pm 7.0\end{array}$ & $\begin{array}{l}56.7 \pm 12.8 \\
43.2 \pm 12.8\end{array}$ & $\begin{array}{l}0.000001 \\
0.000001\end{array}$ \\
\hline
\end{tabular}

Table 2. Comparison of seminal parameters analyzed before and after semen processing through discontinuous density gradient centrifugation.

\begin{tabular}{|l|c|c|c|}
\hline Isolate $\mathbf{( n = 4 1 )}$ & $\begin{array}{c}\text { Before semen processing } \\
\text { Mean } \pm \text { Std Dev }\end{array}$ & $\begin{array}{c}\text { After semen processing } \\
\text { Mean } \pm \text { Std Dev }\end{array}$ & P- value * \\
\hline Concentration (x10\%/ mI) & $81.1 \pm 52.6$ & $64.8 \pm 43.3$ & 0.008457 \\
\hline Motility (\%) & $58.0 \pm 17.7$ & $76.0 \pm 17.4$ & 0.000000 \\
\hline $\begin{array}{l}\text { Morphology (\%) } \\
\text { normal } \\
\text { abnormal }\end{array}$ & $29.6 \pm 11.2$ & $45.3 \pm 15.2$ & 0.000000 \\
\hline $\begin{array}{l}\text { Std Dev=standard deviation } \\
\text { * Dependent Student's t-test with significant difference; } P<0.05\end{array}$ & $54.0 \pm 14.6$ & 0.000000 \\
\hline
\end{tabular}

Table 3. Comparison of the results of sperm washing followed by swim-up and discontinuous density gradient centrifugation before and after semen processing.

\begin{tabular}{|c|c|c|c|c|}
\hline & & $\begin{array}{l}\text { Sperm washing followed } \\
\text { by swim-up }(n=17) \\
\text { Mean } \pm \text { Std Dev }\end{array}$ & $\begin{array}{l}\text { Isolate }(n=41) \\
\text { Mean } \pm \text { Std Dev }\end{array}$ & P-value * \\
\hline \multirow{2}{*}{$\begin{array}{l}\text { Concentration } \\
\left(\times 10^{6} / \mathrm{ml}\right)\end{array}$} & before & $92.5 \pm 56.9$ & $81.1 \pm 52.6$ & 0.4642 \\
\hline & after & $29.0 \pm 28.2$ & $64.8 \pm 43.3$ & 0.0027 \\
\hline \multirow[t]{2}{*}{ Motility (\%) } & before & $52.8 \pm 12.9$ & $58.0 \pm 17.7$ & 0.2748 \\
\hline & after & $76.5 \pm 18.5$ & $76.0 \pm 17.4$ & 0.9285 \\
\hline \multirow{3}{*}{$\begin{array}{l}\text { Morphology (\%) } \\
\text { normal }\end{array}$} & & & & \\
\hline & before & $29.8 \pm 7.0$ & $29.6 \pm 11.2$ & 0.9450 \\
\hline & after & $56.7 \pm 12.8$ & $45,3 \pm 15.2$ & 0.0095 \\
\hline \multirow[t]{2}{*}{ abnormal } & before & $70.1 \pm 7.0$ & $70.3 \pm 11.2$ & 0.9450 \\
\hline & after & $43.2 \pm 12.8$ & $54.0 \pm 14.6$ & 0.0106 \\
\hline
\end{tabular}

which technique was superior (Boomsma et al. 2007).

Both sperm capacitation methods used in the present study recovered the minimum amounts of sperm required to perform IUI. Our results agree with the results presented by Khalil et al. (2001). The comparison of the two techniques before sperm preparation revealed that none was favored.

After sperm preparation, lower sperm concentration recovery rates and higher morphology recovery rates were observed in sperm washing followed by swim-up than in discontinuous density gradient centrifugation. These findings indicate an increase in sperm selection wherein concentration decreases as morphologically normal spermatozoa are selected, as seen when the two methods were compared for sperm morphology after preparation, revealing that sperm washing followed by swim-up had better results in normal sperm recovery rates than discontinuous density gradient centrifugation $(P=0.0095)$. Our results are in agreement with the results reported by Fraczek et al. (2004), which showed that sperm selected by swimup presented slightly better viability and morphology than cells isolated by discontinuous density gradient centrifugation. In contrast, Prakash et al. (1998) and Hammadeh et al. (2001) found a higher percentage of morphologically normal sperm after using discontinuous density gradient centrifugation. Xue et al. (2014) compared swim-up and discontinuous density gradient centrifugation in patients with teratozoospermia, and their results suggested that enrichment with normal morphology spermatozoa with intact DNA could be achieved through swim-up or discontinuous density gradient centrifugation, when compared to 
unprocessed semen. Other authors have reported similar results ( $\mathrm{Ng}$ et al., 1992; Hammadeh et al., 2001; Chiamchanya et al., 2010). Furthermore, Xue et al. (2014) also observed that swim-up produced a higher sperm deformity rate than discontinuous density gradient centrifugation, which produced more favorable results. These findings echo with the data reported by Hammadeh et al. (2001) and Jayaraman et al. (2012), who described a higher percentage of morphologically normal spermatozoa using discontinuous density gradient centrifugation in infertile and teratozoospermic patients, respectively. However, Borges et al. (2013) found no significant differences in the percentages of morphologically normal sperm cells between the two methods in infertile patients. This may be explained by differences in the centrifugation medium used or the type of patients selected in each study.

In the present study, when we compared the results from the two techniques after semen processing, discontinuous density gradient centrifugation had better concentration recovery rates than sperm washing followed by swim-up; our results concord with Huang et al. (1996). Sperm concentrations below $5 \times 10^{6}$ have been associated with low counts of sperm with normal morphology, and impaired sperm motility in such cases is suggestive of lower chances of fertilization (Wainer et al., 2004). According to Karamahmutoglu et al. (2014), discontinuous density gradient centrifugation significantly improves clinical outcomes (pregnancy rates) in IUI cycles of couples with unexplained subfertility and favorable seminal parameters when compared to the swim-up technique. The two techniques produce similar clinical outcomes for subfertile men. This confirms that the efficacy of discontinuous density gradient centrifugation is more pronounced in couples with unexplained subfertility, in which seminal parameters are within the normal range. In addition, Karamahmutoglu et al. (2014), showed that, contrary to their own results, in situations where neither method was superior in male factor patients, it has been accepted that discontinuous density gradient centrifugation is the preferred method in IUI cycles in which sperm counts are low or sperm motility is impaired. Despite evidence suggesting increased pregnancy rates with discontinuous density gradient centrifugation, the authors concluded that their results did not reveal the evidence to support a possible explanatory mechanism.

Motility increased significantly and similarly with both techniques when they were analyzed separately; when compared to each other, no statistically significant differences were found. According to Huang et al. (1996), discontinuous density gradient centrifugation allows for greater recovery of highly motile sperm to perform IUI. However, Posada et al. (2005) showed that the increased clinical pregnancy rates associated with the use of swim-up could be explained by a significantly greater number of totally motile spermatozoa in prewash and post-wash semen samples, when compared to discontinuous density gradient centrifugation. According to some authors, recovery rates of totally motile, progressively motile, and viable sperm cells were higher after with discontinuous density gradient centrifugation than with swim-up (Ding et al., 2000; Ricci et al., 2009). However, Chantler et al. (2004) observed that the ratio of fast sperm was enhanced with the swim-up method.

Another point to be considered is the presence of leukocytes that produce reactive oxygen species (ROS), which change the sperm membrane and might cause sperm DNA fragmentation (Aitken \& Clarkson, 1987; Aitken \& Clarkson, 1988; Griveau \& Lannou, 1994; Aitken et al., 1995). According to Li et al. (2012), the two techniques produce decreased rates of sperm DNA fragmentation after semen processing, while other studies have shown that discontinuous density gradient centrif- ugation efficiently selects sperm with better DNA and chromatin structures (i.e., sperm with greater fertilization potential) (Sakkas et al., 2000; Morrell, 2004).

During the washing procedures, sperm and other components present in the ejaculate settle down, excluding only the seminal plasma, which increases the contact of spermatozoa with other cells that produce ROS during the incubation period for the swim-up technique (Ford, 1990). The discontinuous density gradient centrifugation method already mechanically separates leukocytes, debris, and most of the dead sperm cells. The highly functional sperm selected are exposed to cells that produce ROS for a shorter time period in discontinuous density gradient centrifugation than in swim-up (Jayaraman et al., 2012). Sperm recovery by discontinuous density gradient centrifugation separates motile sperm from other cells, and is a good alternative for cases with higher concentrations of leukocytes, debris, and dead sperm.

Both techniques have advantages and disadvantages. Sperm washing followed by swim-up is an inexpensive technique in laboratory settings, and takes approximately twice as long as discontinuous density gradient centrifugation; furthermore, while the samples are incubated the lab technician can perform other tasks in the laboratory. When using the swim-up technique, sperm quality (i.e., semen with higher sperm concentration) should be taken into account (Henkel \& Schill, 2003). However, this method presents some disadvantages: it is restricted to ejaculates with high sperm count and motility; it is a low-yield technique; spermatozoa can be massively damaged by ROS; and there is a significant decrease in the percentage of normally chromatin-condensed spermatozoa (Henkel \& Schill, 2003). The swim-up is a more sensitive technique, in that it has a greater number of factors that may influence the results. Technicians should be specially trained on this technique, so that the final result is the best possible (Neves, 1991).

Discontinuous density gradient centrifugation is easy to perform and yields higher sperm concentrations with less preparation time (approximately 55 minutes). It also has fewer critical points sensitive to error during preparation, as well as higher levels of effectiveness. However, during discontinuous density gradient centrifugation the technician stays in the lab for longer uninterrupted stretches of time from the beginning to the end of the procedure, thus preventing him/her to perform other tasks in the laboratory. According to Henkel \& Schill (2003), the disadvantages are that the production of good interphases between the different media is a little more time-consuming, and a bit more expensive.

Another important factor relates to the costs of the two techniques. A better-equipped and prepared laboratory is required to perform sperm washing followed by swim-up, when compared to discontinuous density gradient centrifugation. This can be an important factor when choosing a semen processing technique. If one chooses to consider only the cost of the materials used during each method, discontinuous density gradient centrifugation is more expensive, as also observed by Neves (1991).

\section{CONCLUSION}

In conclusion, discontinuous density gradient centrifugation presents good semen concentration, motility, and morphology recovery rates after processing; it is a great option for individuals who do not have high sperm concentration; it is less effective in morphological selection; and produces higher concentration rates than sperm washing followed by swim-up. Sperm washing followed by swimup is indicated for semen with high sperm concentration levels, because it presents a lower concentration recovery rate than discontinuous density gradient centrifugation; however, it does provide for more efficient morphological 
selection, mainly for IVF (Englert et al., 1992; Sapienza et al., 1993). The main limitations of the present study revolve around the small number of patients and the fact that all semen samples were normospermic.

\section{Acknowledgements}

The authors wish to thank the Elsevier language Editing http://webshop.elsevier.com/languageediting/ for revising the English text.

\section{CONFLICT OF INTERESTS}

No conflict of interest have been declared.

\section{Corresponding author:}

Cássio Leão Fácio

Center of Human Reproduction of Sao Jose do Rio Preto

São José do Rio Preto/SP - Brazil

E-mail: cassiolfacio@hotmail.com

\section{REFERENCES}

Aitken RJ, Buckingham DW, Brindle J, Gomez E, Baker HW, Irvine DS. Analysis of sperm movement in relation to the oxidative stress created by leukocytes in washed sperm preparations and seminal plasma. Hum Reprod. 1995;10:2061-71.

Aitken RJ, Clarkson JS. Cellular basis of defective sperm function and its association with the genesis of reactive oxygen species by human spermatozoa. J Reprod Fertil. 1987;81:459-69.

Aitken RJ, Clarkson JS. Significance of reactive oxygen species and antioxidants in defining the efficacy of sperm preparation techniques. J Androl. 1988;9:367-76.

Arny M, Quagliarello J. Semen quality before and after processing by a swim-up method: relationship to outcome of intrauterine insemination. Fertil Steril. 1987;48:643-8.

Barroso G, Chaya M, Bolaños R, Rosado Y, García León F, Ibarrola $E$. Prognostic value on recovery rates for the application of sperm preparation techniques and their evaluation in sperm function. Ginecol Obstet Mex. 2005;73:2218.

Berg U, Brucker C, Berg FD. Effect of motile sperm count after swim-up on outcome of intrauterine insemination. Fertil Steril. 1997;67:747-50.

Boomsma CM, Heineman MJ, Cohlen BJ, Farquhar C. Semen preparation techniques for intrauterine insemination. Cochrane Database Syst Rev. 2007:CD004507.

Borges Jr E, Setti AS, Vingris L, Figueira Rde C, Braga DP, Iaconelli A Jr. Intracytoplasmic morphologically selected sperm injection outcomes: the role of sperm preparation techniques. J Assist Reprod Genet. 2013;30:849-54.

Brasch JG, Rawlins R, Tarchala S, Radwanska E. The relations between total motile sperm count and the success of intrauterine insemination. Fertil Steril. 1994;62:150-4.

Carrell DT, Kuneck PH, Peterson CM, Hatasaka HH, Jones $\mathrm{KP}$, Campbell BF. A randomized, prospective analysis of five sperm preparation techniques before intrauterine insemination of husband sperm. Fertil Steril. 1998;69:122-6.

Chantler E, Abraham-Peskir J, Roberts C. Consistent presence of two normally distributed sperm subpopulations within normozoospermic human se- men: a kinematic study. Int J Androl. 2004;27:350-9.

Chiamchanya C, Kaewnoonual N, Visutakul P, Manochan$\operatorname{tr} \mathrm{S}$, Chaiya J. Comparative study of the effects of three semen preparation media on semen analysis, DNA damage and protamine deficiency, and the correlation between DNA integrity and sperm parameters. Asian J Androl. 2010;12:271-7.

Ding DC, Liou SM, Huang LY, Liu JY, Wu GJ. Effects of four methods of sperm preparation on motion characteristics and nitric oxide concentration in laboratory-prepared oligospermia. Zhonghua Yi Xue Za Zhi (Taipei). 2000;63:8227.

Dodson WC, Haney AF. Controlled ovarian hyperstimulation and intrauterine insemination for treatment of infertility. Fertil Steril. 1991;55:457-67.

Dodson WC, Moessner J, Miller J, Legro RS, Gnatuk CL. A randomized comparison of the methods of sperm preparation for intrauterine insemination. Fertil Steril. 1998; 70:574-5.

Dorjpurev U, Kuwahara A, Yano Y, Taniguchi T, Yamamoto Y, Suto A, Tanaka Y, Matsuzaki T, Yasui T, Irahara M. Effect of semen characteristics on pregnancy rate following intrauterine insemination. J Med Invest. 2011;58:127-33.

Englert $\mathrm{Y}$, Van den Bergh $\mathrm{M}$, Rodesch $\mathrm{C}$, Bertrand $\mathrm{E}$, Biramane J, Legreve A. Comparative auto-controlled study between swim-up and Percoll preparation of fresh semen samples for in-vitro fertilization. Hum Reprod. 1992; 7:399402.

Ford WCL. The role of oxygen free radicals in the pathology of human spermatozoa: Implications of IVF. In: Matson PL, Lieberman BA, eds. Clinical IVF Forum; Current Views in Assisted Reproduction. Manchester University Press, UK; 1990. p.123-39.

Fraczek M, Sanocka D, Kurpisz M. Interaction between leucocytes and human spermatozoa influencing reactive oxygen intermediates release. Int J Androl. 2004;27:69-75.

Griveau JF, Le Lannou D. Effects of antioxidants on human sperm preparation techniques. Int J Androl. 1994;17:22531.

Hammadeh ME, Kühnen A, Amer AS, Rosenbaum $P$, Schmidt W. Comparison of sperm preparation methods: effect on chromatin and morphology recovery rates and their consequences on the clinical outcome after in vitro fertilization embryo transfer. Int J Androl. 2001;24:360-8.

Henkel RR, Schill WB. Sperm preparation for ART. Reprod Biol Endocrinol. 2003; 1: 108.

Huang HY, Lee CL, Lai YM, Change MY, Wang HS, Change SY, Soong YK. The impact of the total motile sperm count on the success of intrauterine insemination with husband's spermatozoa. J Assist Reprod Genet. 1996;13:56-63.

Jayaraman V, Upadhya D, Narayan PK, Adiga SK. Sperm processing by swim-up and density gradient is effective in elimination of sperm with DNA damage. J Assist Reprod Genet. 2012;29:557-63.

Karamahmutoglu $\mathrm{H}$, Erdem A, Erdem M, Mutlu MF, Bozkurt $\mathrm{N}$, Oktem M, Ercan DD, Gumuslu S. The gradient technique improves success rates in intrauterine insemination cycles 
of unexplained subfertile couples when compared to swim up technique; a prospective randomized study. J Assist Reprod Genet. 2014;31:1139-45.

Khalil MR, Rasmussen PE, Erb K, Laursen SB, Rex S, Westergaard LG. Homologous intrauterine insemination. An evaluation of prognostic factors based on a review of 2473 cycles. Acta Obstet Gynecol Scand. 2001;80:74-81.

Li Z, Zhou Y, Liu R, Lin H, Liu W, Xiao W, Lin Q. Effects of semen processing on the generation of reactive oxygen species and mitochondrial membrane potential of human spermatozoa. Andrologia. 2012; 44:157-63.

Morrell JM, Moffatt O, Sakkas D, Manicardi GC, Bizzaro D, Tomlinson M, Nilsson H, Holmes PV. Reduced senescence and retained nuclear DNA integrity in human spermatozoa prepared by density gradient centrifugation. J Assist Reprod Genet. 2004;21:217-22.

Neves PA. Comparação entre duas técnicas de capacitação de espermatozoides para reprodução assistida [thesis]. Campinas: Universidade Estadual de Campinas; 1991.

Ng FL, Liu DY, Baker HW. Comparison of Percoll, mini-Percoll and swim-up methods of sperm preparation from abnormal semen samples. Hum Reprod. 1992;7:261-6.

Paasch U, Grunewald S, Glander HJ. Sperm selection in assisted reproductive techniques. Soc Reprod Fertil Suppl. 2007;65:515-25.

Pasqualotto FF. Investigation and assisted reproduction in the treatment of male infertility. Rev Bras Ginecol Obstet. 2007; 29: 103-12.

Posada MN, Azuero AM, Arango AM, Raigosa GC, Cano JF, Perez AL. Sperm washing with swim-up versus gradients in intrauterine insemination (IUI): results of a prospective randomized study comparing pregnancy rates and costs. Fertil Steril. 2005; 84:s361

Prakash P, Leykin L, Chen Z, Toth T, Sayegh R, Schiff I, Isaacson K. Preparation by differential gradient centrifugation is better than swim-up in selecting sperm with normal morphology (strict criteria). Fertil Steril. 1998;69:722-6.
Ricci G, Perticarari S, Boscolo R, Montico M, Guaschino S, Presani G. Semen preparation methods and sperm apoptosis: swim-up versus gradient-density centrifugation technique. Fertil Steril. 2009;91:632-8.

Sakkas D, Manicardi GC, Tomlinson M, Mandrioli M, Bizzaro $D$, Bianchi PG, Bianchi U. The use of two density gradient centrifugation techniques and the swim-up method to separate spermatozoa with chromatin and nuclear DNA anomalies. Hum Reprod. 2000;15: 1112-6.

Sapienza F, Verheyen G, Tournaye H, Janssens R, Pletincx I, Derde M, Van Steirteghem A. An auto-controlled study in in-vitro fertilization reveals the benefit of Percoll centrifugation to swim-up in the preparation of poor-quality semen. Hum Reprod. 1993;8:1856-62.

Scemama H, Salat-Baroux J, Antoine JM, Saada H. Value of controlled hyperstimulation in intrauterine inseminations. Contracept Fertil Sex. 1995;23:37-44.

Wainer $R$, Albert $M$, Dorion A, Bailly $M$, Bergère $M$, Lombroso R, Gombault M, Selva J. Influence of the number of motile spermatozoa inseminated and of their morphology on the success of intrauterine insemination. Hum Reprod. 2004; 19:2060-5.

WHO - World Health Organization. WHO laboratory manual for the examination of human semen and sperm-cervical mucus interaction. Cambridge: Cambridge University Press; 1999.

Xue X, Wang WS, Shi JZ, Zhang SL, Zhao WQ, Shi WH, Guo BZ, Qin Z. Efficacy of swim-up versus density gradient centrifugation in improving sperm deformity rate and DNA fragmentation index in semen samples from teratozoospermic patients. J Assist Reprod Genet. 2014;31:1161-6.

Yoshida M, Kawano N, Yoshida K. Control of sperm motility and fertility: diverse factors and common mechanisms. Cell Mol Life Sci. 2008;65:3446-57.

Zhao Y, Vlahos N, Wyncott D, Petrella C, Garcia J, Zacur H, Wallach EE. Impact of semen characteristics on the success of intrauterine insemination. J Assist Reprod Genet. 2004;21:143-8. 